\title{
Ethical Consideration of Euthanasia for People Diagnosed with Dementia
}

\author{
Baljit Kaur Gill* \\ Associate Professor, School of Nursing and Health Studies, The Open University of Hong Kong, Hong Kong
}

*Corresponding author: Baljit Kaur Gill, Associate Professor, School of Nursing and Health Studies, The Open University of Hong Kong, Hong Kong.

Received Date: March 03, 2020

Published Date: March 17, 2020

\begin{abstract}
Euthanasia is a deliberative intervention embracing the determination of ending a life so as to alleviate awkward and unbearable suffering. It is only taken into consideration when by the person is mentally competent. With the advance of medical knowledge, there is a better understanding of the prognosis of dementia. Individuals' diagnosed with dementia often expresses that they do not want to be a burden to their family members as the disease progress and often expresses they want to end their life before they are not able to take care of themselves. The aim of this paper is to critique the feasibility and ethical considerations of euthanasia among individuals diagnosed with dementia using 'MORAL' ethical decision-making model. Patient (individual diagnosed with dementia), caregiver, nurses and physician should be included in order to have a deeper understanding of the euthanasia ethical dilemma. Different options such as treatment available, type and strategies appropriate for the dementia case, appropriate ethical theories in the dementia case (beneficence and autonomy). Then, it is essential to review and "look back" at the case. In order to enhance the possibilities of euthanasia among individual with dementia, advance directives on euthanasia could be an option.
\end{abstract}

Keywords: Euthanasia; Dementia; Moral ethical model

\section{Introduction}

Euthanasia is a subject of much controversy worldwide in that it touches on different parties' rights and responsibilities as well as diverse ethical concerns. Euthanasia is a deliberative intervention embracing the determination of ending a life to alleviate awkward and unbearable suffering [1,2]. Euthanasia is embraced and legislated in some countries, including Dutch, Belgium, Luxembourg, Colombia, Canada, several States in America [2]. In Dutch, patients who have a desire for euthanasia must be proved by two physicians that they are under unbearable suffering with no anticipation of improvement which is no need to be linked up with terminal diseases and not restricted to physical illnesses, and they must be competent, well-informed with sufficient information which ensures that their requests are constructed on a voluntary basis and after a careful deliberation [2,3].

Desires and concerns over euthanasia among patients being diagnosed as dementia have arisen in recent years. However, there is limited evidence that whether the request of euthanasia made by | dementia patients should be accepted and whether it is the best option for them. Therefore, ethical dilemma emerged ascribed to the difference between patients' desired goal and current mainstream situation worldwide. In order to explore and resolve the ethical dilemma of this controversial issue, this paper had used Crisham's 'MORAL' model as a framework for guiding the analysis of ethical dilemma on individuals diagnosed with dementia patients and future recommendation on the issue was discussed.

\section{Dementia}

Dementia refers to an incurable, progressing deteriorating and chronic syndrome with a gradual decline of cognitive functions, including memory loss and impaired judgement which impedes activities of daily living and quality of life [4,5]. Deterioration in emotional control, personality change and behavioral problems are also commonly seen in patients with dementia $[4,6]$. There are several types of dementia, which Alzheimer's disease which is the most common form of dementia, vascular dementia, dementia with Lewy 
bodies, Parkinson's dementia, frontotemporal dementia, etc. [5,6]. According to Boyd 2014 [4], course of dementia can be classified into three stages: Mild stage is manifested by loss of memory, mood swing, diminished judgement and aphasia; Moderate stage is characterized by inability to retain new knowledge, behavioral change such as aggression and agitation; In severe stage, patients will demonstrate motor disturbance like apraxia, immobility, inability to perform activities of daily living and inability to communicate.

\section{Different Types and Strategies of Euthanasia}

Euthanasia can be categorized into three types namely

1) Voluntary euthanasia arises based on patients' request to die on his/her own free will (British Broadcasting Corporation, 2014a) [7,8].

2) Involuntary euthanasia is the one without the patient's request and consent, or even when patient expressed intention to life, yet euthanasia is enacted according to the physician's decision (British Broadcasting Corporation, 2014a; Shea, 2010) [7-9].

3) Nonvoluntary euthanasia is when patients are unable to give informed consent, such as patients who are unconscious or in a persistent vegetative state, children who are not mentally or emotionally competent (British Broadcasting Corporation, 2014a; Jones, 2011) $[7,8,10]$.

Euthanasia can be carried out in either active or passive way. Active euthanasia occurs when a patient's death is directly brought by a deliberate act by a facilitator [1]. While passive euthanasia refers to patient's death is obliquely brought by an exclusion like withholding or withdrawing life-sustaining treatments such as surgery or apparatus such as respirator and Ryles Tube, for the maintenance of the patient's life [1].

\section{'MORAL' Ethical Decision-Making Model}

Gaining a thorough understanding and getting into a deep analysis, an ethical decision-making model called 'MORAL' Crisham, 1985 [11] was used in the decision-making on euthanasia request in persons who have been diagnosed as dementia. According to Crisham1985 [11], this model consists of five parts: 'M' for Massage the Dilemma: In order to evolve a thorough and ample understanding of the issue, it calls for collecting relevant and vital data such as values, concerns and interests between parties involved and point out conflicting contexts [8]; 'O' for Outline Options: It entails impartial and systemic consideration and reflection of every possible choice which helps solve the conflicts; 'R' for Review Criteria and Resolve: This step emphasized on recognizing moral criteria and ethical principles that help generate moral judgement; 'A' for Affirm Position and Act: It requires to execute the planned actions; 'L' for Look Back: it highlights evaluations on change of person's perceptions, comprehensiveness of options, effectiveness and practicability of actions implemented and whether the conflicts are resolved [12].

\section{M: Massage the Dilemma}

This process aims to identify whose interests are involved in the conflict, define the dilemma from and consider options of the all the major parties' perspectives or values [13]. This includes patients, caregiver, nurses and physicians.

\section{Patients}

Dementia is a medical condition that leading to gradual loss of abilities over a lengthy period of time [14]. It has impacts on a patient's social, psychological, emotional and economic aspects. By the knowledge that dementia is a progressive disease that the condition will get worse, typically involving loss of memory, mood and behaviours changes and deterioration with cognitive functions [14], patients considered the state of advanced dementia is devoid of dignity and unbearable [3]. Patients do not want to be obliged to undergo the future decline and suffering. This is a main reason for those who opt for euthanasia in the early stage of the disease [3].

There are social factors affecting the patient's decision on euthanasia. Patients might be directly or indirectly pressured into requesting euthanasia by caregivers who are no longer able or willing to carry the burden of care; or they might request euthanasia in order to alleviate the burden of their care for their caregivers [15]. Besides, religious perspectives on euthanasia would be a consideration factor. Most of the major religious organizations, including Christianity, Buddhism are opposed to euthanasia as treated their lives were a gift from God and they prohibited intentional killing [16]. Therefore, those believers would not perform euthanasia.

\section{Caregivers}

Caregivers suffer from tremendous physical and psychological stress. Caregivers sacrifice their own needs and well-being to provide care for dementia patient, including managing changing demands and unexpected behaviours [17]. Patients may wish to not only relieve their own suffering but also the suffering of the people they love [15]. Supporting euthanasia to relieve patients and the caregiver suffering. However, it is noted that not all caregivers had distress and burden. Some of them reported satisfaction with providing care, such as feeling needed and useful, developing appreciation of life and strengthen the relationship between them and patients [17]. Those caregivers might object to dementia patient's request for euthanasia.

\section{Physician}

Health care providers play an important role in euthanasia. Physicians are the gatekeeper as their duty is to sign approval for euthanasia of patients, so they face high psychological stress. In the Netherlands, where euthanasia is legal, nurse's role includes registering the request, making the decision, assisting the euthanasia, and aftercare [18]. Nevertheless, euthanasia may be seen as intentionally hasten the end of life in some of the medical professional views [19]. This is because both specialists Code of Professional Conduct stress on do minimize harm to patients, so performing eu- 
thanasia should be the last resort if no alternative, such as palliative care is available $[20,21]$. However, it is crucial that both specialists acknowledge that patients are fellow human beings who are worthy of dignity and respect [22].

\section{0: Outlines options}

In the second step of the model, it requires to outline options including those are conflicting [13]. This stage is designed only for considering options and not for making a final decision. There are three options for this ethical dilemma by considering general ethics principles.

\section{Option one: continuing medical treatment}

The first option is to continue pharmacological treatment and non-pharmacological methods for patients with dementia. Pharmacological, there are medications that could stabilize the behavioral and psychological symptoms of dementia. For example, antidepressants, antipsychotics, mood stabilizers and benzodiazepine [23]. While the progression of memory deterioration could be alleviated by donepezil, rivastigmine, galantamine [24]. For non-pharmacological therapy, doll therapy could be provided to enhance dementia patients' emotional management. Doll is provided to patients and they are encouraged to interact with the doll, which calm patients' mood and enhance communication abilities by stimulating affective topic of motherhood and caregiving [25]. As a result, it could increase the general well-being, improve dietary intake, promote interpersonal engagement and reduce the episodes of distress [26]. Reminiscence therapy could be also provided to alleviate the memory deterioration of dementia patients. The therapy is based on discussion and sharing about the past experiences and events with another person or a group. During the therapy, various supporting materials are involved such as video, pictures or life story books. Since the remote memory remains intact until the later stages of dementia, it can be used as a form of communication with dementia patients [27].

\section{Option two: passive euthanasia}

The second option is passive euthanasia. Healthcare workers do not continue to provide life-prolonging medical treatment so as to bring about patients' death [28,29]. Passive euthanasia commonly includes withholding or withdrawing the following life-sustaining treatments:

Cardiopulmonary resuscitation: Cardiac disease is one of the leading causes of death for patients with dementia [30]. When cardiac arrest is anticipated, cardiopulmonary resuscitation can indicate the opportunity for survival [31]. However, in passive euthanasia, healthcare professionals will not perform cardiopulmonary resuscitation when cardiac arrest occurs.

Artificial ventilation (ventilator): Artificial ventilation avoids deaths from choking, a common incident of elderly people [32]. In passive euthanasia, healthcare professionals will not provide air to patients who are breathlessness or cannot breathe sufficiently.
Blood products (transfusion, blood platelet, blood plasma): Blood products are a lifesaving measure to repair blood or plasma volume during surgeries and clinical treatments for severe injuries or diseases for older adults [33]. In passive euthanasia, healthcare professionals will not provide blood products to patients whose blood must be replaced.

Pacemakers and vasopressors: Pacemakers and vasopressors are also commonly use in dementia patients with cardiac disease. For instance, cardiac pacemakers are used for patients with cardiac arrhythmias and vasopressors such as adrenaline are powerful drugs that can elevate the arterial pressure when anaphylaxis and cardiac arrest [34]. In passive euthanasia, healthcare professionals will not provide pacemakers and vasopressors to patients that cardiac function deteriorated.

Antibiotics: Antibiotics are widely used to treat pneumonia, which is a common disease among patients with advanced dementia, especially at the end of life, such as aspiration pneumonia [35]. In passive euthanasia, healthcare professionals will not provide antibiotics for patients with infectious disease.

Artificial nutrition and hydration (nasogastric tube): Deterioration of swallowing ability leads to dehydration and losing weight in advanced dementia patients and artificial nutrition and hydration are used to prevent life-threatening consequences [36]. In passive euthanasia, healthcare professionals will not deliver artificial nutrition and hydration to patients even if their oral intake of food and liquids is not possible anymore.

\section{Option Three: Active Euthanasia}

The last option that nurse may consider is active euthanasia. Physicians terminate patients' life so as to relieve them with unbearable suffering with no prospect of improvement [37]. Every step of the active euthanasia must be performed by a physician, for ensuring that all the due care criteria have been met [38]. With reference to Dutch guidelines, active euthanasia proceeds in a sequence of medical procedures [39]. First, premedication of sedatives like midazolam would be used [40]. After that, the physicians will inject sodium thiopental as an intravenous coma-inducing substance. Meanwhile, health care workers are obligated to ensure that the depth of coma of patients is deep enough, so that they cannot sense the effects of muscle relaxants. Lastly, an alcuronium chloride or pancuronium bromide as an intravenous muscle relaxant would be administered to cause paralysis and end their life [41]. If the physicians have used unlisted substance and not suggested dose, they must provide convincing arguments to support their behavior. Besides, it is recommended to inform the patients and family members about the effects of the substance before the injection.

\section{R: Review Criteria and Resolve}

This part focuses on recognizing the moral criteria and ethical principles of the mentioned three options. This can facilitate to make a moral judgment on each option which generates the most 
suitable option through comparisons between options. There are two ethical principles involved, which are beneficence and autonomy.

\section{Beneficence}

Beneficence is balancing the benefits of treatment against the risks and costs involved [42] and means always doing good for patients [43]. Beneficence acts range from multiple dimensions, including physical, psychological and spiritual aspects, instead of solely reduction in physical suffering [44]. Euthanasia is directly intented killing the patients, so it is undoubtedly doing harm to patients and does not follow the principle of beneficence [45].

\section{Autonomy}

Autonomy is defined as allowing the patients to make his or her choice. This can be expressed when health care providers respect and permit patient's decision on medical treatments including euthanasia [46]. However, the concept of autonomy requires individual to be fully conscious and informed about the situation, and mentally capable to think, decide, and act on such thought freely and independently [46].

\section{Reviewing Option One: Continuing Medical Treatment}

Continuing medical treatment fulfils only the ethical principle of beneficence, but not autonomy. This option is beneficial to the patients, family members and friends. The benefits for patients are the reduction of the deterioration and promote health status. As dementia would have worsened cognitive function, patient's condition could be stabilized, and further deterioration of the condition could be slowed down by receiving medication and care [47]. Besides, medical treatment and therapies could enhance the well-being of patients either physically or mentally [26]. For example, doll therapy is effective in promoting demented patients' sense of happiness [25]. Other psychological intervention may also assist patients in discovering the meaning of their life so as to address patients' wish of euthanasia due to the intolerable suffering [44]. This option could also benefit family members and friends because patients could be a source of emotional support to them [48]. The dementia patients might be the significant others of his/her family members and friends so they might establish close relationships. Continuing medical treatment could keep patients alive such that family members and friends could keep bonding with patients. Maintaining such bonding could create new memorable moments and be a source of support to the family members and friends. Yet, the principle of autonomy is violated because this option does not act according to patients' wish. Patients want to terminate their lives so as to reduce the awkward and not bearable suffering from dementia [2]. They desire to end their lives with dignity and respect. Whereas, healthcare providers do not permit so. Medical treatments and interventions keep providing to patients and their lives are prolonged which it is not aligned with patients' preferences.

\section{Reviewing Option Two: Passive Euthanasia}

Under this option, the ethical principle of autonomy is respected but there is no beneficence. Giving permission of euthanasia facilitates demented patients to end their intolerable suffering early so that patient's wish is followed. However, it violates the principle of beneficence which withholding life-sustaining medical treatment leads to progressive and continuous long-term suffering until the end of patient life. Medication and life-sustaining assistant given could result in considerable suffering. For example, life-sustaining intervention, such as ventilator and nasogastric tube would be removed. Removal of ventilator could induce breathing difficulties and hence fear, while starving to death is not beneficence. Moreover, discontinuing active medications such as antibiotics is one of the examples in passive euthanasia. Since pneumonia is common among dementia patients, withholding antibiotics would cause worsening infection and intensify patients suffering before death [35]. Thus, passive euthanasia is not beneficence even it ensures patients' desire of death can be accomplished.

\section{Reviewing Option Three: Active Euthanasia}

The ethical principle of autonomy could be demonstrated in active euthanasia but not beneficence. Patient's early decision on euthanasia is respected which assures patients' autonomy. Nevertheless, even it follows the principle of autonomy, it still directly leads to the death of patient through the mean of injection of medication in order to induce coma, inhibit patients' respiratory center and ultimately death. Intentionally ending patients' life is still considered as a harm to patient as defined. Thus, active euthanasia is contradictory with the principle of beneficence, even it allows the patients to terminate intolerable suffering.

\section{Making Decision on the Best Option}

Reviewing three available options, ethical struggle exists between two seemingly equally weighted ethical principles, which are beneficence and autonomy. The acceptance of active and passive euthanasia respects the determination of patient (autonomy), while imposing harm to patients (not beneficence). On the contrary, continue treatment promotes the well-being of patients (beneficence), while not act in patient will (not autonomy). Indeed, the best decision among three possible solution could be ascertained via thorough investigation.

\section{Capability of Autonomy}

Personal autonomy is often perceived as the most important ethical principle that should be considered [49]. Deciding a solution that could consistent with patients' will is crucial. Accepting the request of active and passive euthanasia is an expression of autonomy as patient's decision on medical treatments is valued. However, such concept of self-determination may not be applicable for patient with dementia. The concept of autonomy requires individual to be fully conscious and informed about situation, and mentally capable to think, decide, and act on such thought freely and 
independently [46]. Applying a medical decision-making capacity model developed by Grisso and Appelbaum 1998 [50], it formulates four criteria for decision-making capability, namely

I. the ability to communicate a choice.

II. understand treatment-related information.

III. appreciate medical consequences of own situation.

IV. logical reasoning to about treatment options.

Patients with dementia, nonetheless, might not able to fulfill such criteria. With reference to the disease progress, dementia can lead to cognitive decline in various extent, including impaired short-term memory, language difficulty, trouble understanding and poor judgment [51]. The above impairments could adversely affect patients' ability to understand or express speech and make appropriate judgement for their treatment options. As a result, dementia patients may not fulfill the above criteria and hence indicating an inadequacy of decision-making capability.

The detrimental effect of cognitive impairments is irreversible throughout the entire pathological progress [52]. It is not surprising that patients with middle to late stage dementia will be unable to understand and evaluate the information necessary to make these requests [1]. Indeed, even mild stage dementia can have substantial effects on decisional capacity [53]. It was because premorbid mild cognitive impairment usually begins three to four years precede formally diagnosis of dementia [54]. A systematic review supplemented that majority of participants with dementia and cognitive impairment, ranging from mild to severe, were incapable of making their own health-related decisions like advance care plans or advance directives [55]. Therefore, neither passive euthanasia nor active euthanasia that based on patient's autonomy should be taken precedence in concern as dementia patients might not possess the capacity to fully understand the situation and express informed consent before the request of euthanasia being made.

\section{Beneficence Outweigh Autonomy}

As the mental competency of dementia patients are jeopardized regardless of stage, the consideration of option would be shifted from autonomy to beneficence. According to the ethical principle of beneficence, health care workers are obligated to provide care in the best interest of patients [56]. Beneficent and safe care were morally and legally demanded by the professional role, which have been emphasized in many code of ethics and professional conduct. For example, the Nursing Council of Hong Kong (2015) stated that "Nurses raise objection to practices that compromise safe and appropriate care (p.10)". If there are foreseeable harm or consequences for compliance with patient's euthanasia request, such as affecting the significant interests of patient, patient's family and friends, medical staff is not obligated to perform [57]. Therefore, following patient's request for either active or passive euthanasia should not be accepted as it is posing threat to physical health of patients and psychological health of significant others.
For instance, patients would suffer from physical damage during euthanasia and family members might suffer from bereavement. Performing treatment that causing harm to patient or family member cause violation to our professional nursing conduct, and hence should not be promoted. Instead, providing ongoing treatment with by health care workers is more beneficent. Not only can treatment reduce deterioration and prolong meaningful life of patients, but it also allows significant others to treasure the remaining time with patients. In sum, with reference to the analysis of three options along with ethical principles, the best option to proceed would be continued provision of medication and care to dementia patients.

\section{A: Affirm position and act}

In this process, continuing provision of medication and care to dementia patients should be implemented as the selected option to resolve the ethical struggle. Healthcare providers should follow through on the case-specific plan of action. The actions included the pharmacological treatment like antipsychotics and psychological intervention such as reminiscence therapy which are beneficial to patients' health both physically and mentally.

\section{L: Look back}

In this step, health care providers complete an evaluation to determine whether the ethical struggle have been resolved. Furthermore, patients' response and their concerns are needed to be evaluated such as whether the ethical principle would be fulfilled. Through evaluation, improvements could be made such that a better decision could be formulated currently and, in the future [58].

\section{Recommendation}

With reference to the option of continue treatment selected above, it is convinced that this option only fulfills the principle of beneficence but not autonomy. Although it is beneficence to patients because of maintaining their health status, autonomy is violated as demented patients are regarded as incompetent to make decisions such that the requests for euthanasia are considered as not autonomous. By means of that, Advance Euthanasia Directives would be recommended. Drafting Advance Euthanasia Directives before dementia occurs on one hand could secure an autonomous decision with decision-making capacity. On the other hand, it could also relieve unbearable suffering (beneficence) according to patients' decision. Therefore, AED would be a preferable option comparing to the continue treatment among the at-risk individuals for dementia.

\section{Introduction of Advance Euthanasia Directives}

Advance Euthanasia Directive (AED) is a special type of advance directives for executing euthanasia. It can be defined as statements made before individual proceeds to a stage of incapacity in decision-making process so as to allow the individual to provide directions for future care and medical decision. It can be either an oral or written form [59]. Preparing and drafting an AED could be one of 
the methods for patients to gain a sense of control over future [60]. Besides, AED could empower patients to relieve suffering at the severe illness by making AED to avoid unwanted medical treatment like nasogastric tube insertion [61].

\section{Autonomy in Advance Euthanasia Directive}

The autonomy of patients can be guaranteed by the means of Advance Euthanasia Directive. AED is one of the efforts to respect for autonomy of patients because it is a tool for them to have self-determination about their current and future life [62]. It is believed that AED can help patients give voice to their values and preferences. The amendment of AED could be made whenever patient requires, in the premise that patients are still mentally competence in decision making assessed by physicians [63]. The capability of autonomy of patients without dementia in AED would be explained by applying the medical decision-making model [50] mentioned above.

For the ability to communicate, people with dementia might encounter the communication problems such as language or speaking difficulties whereas people without dementia might not [64]. Thus, the formulation of AED should be made with effective communication before people are diagnosed with dementia. In real cases, one of the major problems in AED is that the trigger point for implementing euthanasia is too broad and ambiguous among demented patients. Through meaningful communication between patient and physician, it helps physicians and relatives understanding patients' values and preferences. Physicians hence could be more precise to determine whether the patients reach the triggering point of unbearable suffering [65]. In short, people without dementia can establish a meaningful communication unlike the demented, hence the trigger point could be correctly interpreted based on patients' preferences such that autonomy of patients could be respected.

With reference to another three criteria including understanding treatment-related information, appreciating medical consequences of own situation and logical reasoning to about treatment options, it focuses on making an appropriate judgment on AED. During the preparation phase of AED, patients are required to imagine their quality of future life in order to weight the good and bad by themselves, for example, anticipating burden of future health deterioration. However, demented patients may not be capable to do so due to impairment in cognitive function [54]. Besides, people without dementia is able to evaluate their current medical situations such as anticipated illnesses based on the information provided by the healthcare providers. In contrast, demented patient is not fully competent to understand the relevant information about their situations and implications of the decision [66]. Therefore, people without dementia have a decision-making capacity to generate a valid medical choice so that the AED made by them is argued as autonomous decision.

\section{Beneficence in Advance Euthanasia Directive}

AED is known as beneficence for patients because its ultimate goal is death which could be a method to relieve them from unbear- able suffering. The suffering of patients often depends on fear of further decline and negative impact on their autonomy and dignity [67]. Taking a case study from Dutch as example, a woman aged 64 years old was diagnosed as dementia. She did not want to have experiences of double incontinence, nasogastric tube insertion and living an old-age home in further deterioration. The above concerns were meaning unbearable suffering for her. Therefore, she took $\mathrm{AED}$ as a method for her to relieve from those suffering [68].

\section{Prevention Better Than Cure}

Although continued treatment is deemed as the best option for patients with dementia, establishing an AED precede could be a better alternative for people who are still mentally competence. It is particularly crucial for people who are at-risk of dementia. Oldaged, having family history of dementia, genetics and head trauma are the common risk factors of dementia [69]. Discussing an option of euthanasia with a patient is not the same as encouraging them to take his or her own life, but rather empowering them to make informed decision [70]. Thus, AED should be promoted to the population at risk of dementia, especially in hospital and community.

\section{Hospitals}

In typical geriatric settings, patients with advanced age, trauma and family history of dementia are ubiquitous. Hospitals, in organizational context, could aim at raising the awareness of AED and getting people noticed the issue about AED. It could be achieved via providing information about AED through online platforms, posters and leaflets [71]. While in individual context, physicians are suggested to be engaged in the promotion of AED. As some people may assume that health professionals will initiate discussions automatically when appropriate [55]. Initiative should be taken by the physicians to communicate with the patients at risk of dementia. Information about AED could be provided at a regular means, such as comparing the pros and cons [59]. Nurses are also obligated to discuss and clarify for the unclear details and explore concerns of patients' [70].

\section{Community}

The completion rate of AED among older people is insignificant in the current society (Bradley, Woodman, [72]. One of the contributory factors is that some older people do not actually aware AED exist [55]. Therefore, enhancing public's knowledge and awareness about AED would be essential. To be more specific, nursing homes for elderly is stressed as the main target as numerous of at-risk individuals could be commonly accessed. Information related to the current development and policy of AED could be delivered to enhance the acceptance of AED [73-76].

\section{Conclusion}

The paper has discussed the ethical dilemma of whether request on euthanasia made by dementia patient would be justified. Euthanasia is referred to terminate or shorten life to relieve one suffering. It can be voluntary, involuntary or non-voluntary and can be carried out in active or passive way. Dementia is an overall term 
that describe chronic and progressive deterioration syndrome with gradual decline of cognitive functions, and it is incurable at present.

Crisham's 'MORAL' model was applied to guide for the discussion on the ethical dilemma. 'M' stands for massage the dilemma, that means collect relevant data from patients, caregivers and healthcare providers because their interests are involved in the conflict. ' $\mathrm{O}$ ' is referred to outline options of continued treatments, passive euthanasia and active euthanasia. ' $R$ ' for Review Criteria and Resolve, for the ethical dimension of three options. Providing continued treatment follows the principle of beneficence but there is no autonomy for patients. While both active and passive euthanasia violate beneficence but respect patient's autonomy. Nevertheless, dementia could cause various degree of cognitive impairments to patients. The capability to understanding, think and decide would be affected regardless of stages, affecting the achievement of autonomy completely. Thus, beneficence, rather, should be consider for dementia patients', so continued treatment would be the best option. 'A' means Affirm Position and Act, the stage to implement the selected option. 'L' for Look Back, which refers to evaluations on different aspects of the model to improve the present and future decision making.

Finally, future recommendation would be establishing the Advance Euthanasia Directive before dementia occurs. Making statements before patients become incompetent in decision-making could satisfy both beneficence and autonomy. Patient could make decision on the future planning of care (autonomy) and the triggering point that the deemed that relieve is needed from intolerable suffering (beneficence). Thus, promotion should be made in both hospital and community settings.

Life, comes with an "e" at last, ending of life is inevitable for every person. Choosing the way to end and passing with dignity are the basic right of every person. However, everyone has chance to suffer from dementia, regardless how worthy you are, how famous you are and who you are. In ordering to ensure decision is made under autonomy, planning for future caring should be explicit with written document in state of mentally competence. By making decision precede dementia, the autonomous and beneficial decision of a person could be warranted and respected.

\section{Acknowledgement}

The author, Dr. Baljit Kaur Gill, would like to thank Bobby Gill for the support of writing this paper.

\section{Conflict of Interest}

The author declares that there is no conflict of interest. No funding has been received.

\section{References}

1. Franklin K (2015) Physician-assisted death, dementia, and euthanasia: Using an advanced directive to facilitate the desires of those with impeding memory loss. Idaho Law Review 51(2): 547-574.
2. Lewis P (2015) Assisted dying: What does the law in different countries say?

3. De Beaufort ID, Van De Vathorst S (2016) Dementia and assisted suicide and euthanasia. J Neurol 263(7): 1463-1467.

4. Boyd M (2014) Psychiatric Nursing: Contemporary Practice $\left(5^{\text {th }}\right.$ edn.), Wolters Kluwer Health/Lippincott Williams \& Wilkins, Philadelphia, USA.

5. World Health Organization (2017) Dementia.

6. Engdahl S (2013) Dementia. Greenhaven Press, Detroit, USA.

7. British Broadcasting Corporation (2014) Forms of euthanasia.

8. Ghazal L, Saleem Z, Amlani G (2014a) A Medical Error: To Disclose or Not to Disclose. Journal of Clinical Research \& Bioethics 5(2): 1-3.

9. Shea F (2010) Hurricane Katrina and the legal and bioethical implications of involuntary euthanasia as a component of disaster management in extreme emergency situations. Ann Health Law 19(1): 133-139.

10. Jones D (2011) Is There a Logical Slippery Slope from Voluntary to Non-voluntary Euthanasia? Kennedy Inst Ethics J 21(4): 379-404.

11. Crisham P (1985) Moral: How can I do what's right? Nurs Manage 16(3): $42 \mathrm{~A}-42 \mathrm{~N}$.

12. Ghazal L, Saleem Z, Amlani G (2014b) Resolving ethical dilemma: An application of a theoretical model. Khyber Medical University Journal 6(3): 135-138.

13. Mc Carthy VP, Le Roy BS, Callanan NP (2018) Facilitating the Genetic Counseling Process: Practice-Based Skills ( $2^{\text {nd }}$ edn.), Springer, USA.

14. Alzheimer Europe (2014) Alzheimer Europe Report: Ethical dilemmas faced by carers and people with dementia.

15. Dementia Australia (2011) Planning for the End of Life for People with Dementia.

16. Shuriye AO (2012) Ethical and Religious Analysis on Euthanasia. International Islamic University Malaysia Engineering Journal 12(5): 209211.

17. Tremont G (2011) Family caregiving in dementia. Med Health R I 94(2): 36-38.

18. Inghelbrecht E, Bilsen J, Mortier F, Deliens L (2009) Attitudes of nurses towards euthanasia and towards their role in euthanasia: A nationwide study in Flanders, Belgium. Int J Nurs Stud 46(9): 1209-1218.

19. Seale C (2009) Legalisation of euthanasia or physician-assisted suicide: Survey of doctors' attitudes. Palliat Med 23(3): 205-212.

20. American Nurse Association (2013) Euthanasia, Assisted Suicide, and Aid in Dying.

21. American Medical Association (2018) Euthanasia.

22. Quaghebeur T, Dierckx de Casterlé B, Gastmans C (2009) Nursing and Euthanasia: A Review of Argument-Based Ethics Literature. Nurs Ethics 16(4): 466-486

23. Cerejeira J, Lagarto L, Mukaetova Ladinska EB (2012) Behavioral and Psychological Symptoms of Dementia. Front Neurol 3(73): 1-21.

24. Rodda J, Morgan S, Walker Z (2009) Are cholinesterase inhibitors effective in the management of the behavioral and psychological symptoms of dementia in Alzheimer's disease? A systematic review of randomized, placebo-controlled trials of donepezil, rivastigmine and galantamine. Int Psychogeriatr 21(5): 813-824.

25. Pezzati R, Molteni V, Bani M, Settanta C, Di Maggio, et al. (2014) Can Doll therapy preserve or promote attachment in people with cognitive, behavioral, and emotional problems? A pilot study in institutionalized patients with dementia. Front Psychol 5: 342.

26. Mitchell G, Templeton M (2014) Ethical considerations of doll therapy for people with dementia. Nurs Ethics 21(6): 720-731. 
27. Cotelli M, Manenti R, Zanetti O (2012) Reminiscence therapy in dementia: A review. Maturitas 72(3): 203-205.

28. Khazali M (2017) Mercy Killing. Trukish Online Journal of Design Art and Communication 7: 663-671.

29. Garrard E, Wilkinson S (2005) Passive euthanasia. Journal of Medical Ethics 31(2): 64-68.

30. Arcand M (2015) End-of-life issues in advanced dementia: Part 1: goals of care, decision-making process, and family education. Can Fam Physician 61(4): 330-334.

31. Hospital Authority Clinical Ethics Committee (2016) Advance Care Planning (ACP)? Advance Directives (AD)? Do-Not-Attempt Cardiopulmonary Resuscitation (DNACPR)? Patients and families should know more.

32. Encyclopedia Britannic (2018a) Artificial respiration.

33. Encyclopedia Britannic (2018b) Blood transfusion.

34. Burns SM (2014) AACN essentials of critical care nursing ( $3^{\text {rd }}$ edn.), American Association of Critical-Care Nurses, Washington, USA.

35. Mitchell SL (2015) Clinical practice. Advanced dementia. N Engl J Med 372(26): 2533-2540.

36. Martin C R, Preedy VR (2014) Diet and nutrition in dementia and cognitive decline ( $1^{\text {st }}$ edn.), Cambridge Academic Press, UK.

37. Johnson RR (2016) Moral Permissibility of Active Euthanasia. Unpublished master's thesis, The University of Oslo, Oslo.

38. Gysels M, Evans N, Meñaca A, Andrew E, Toscani F, et al. (2012) Culture and end of life care: a scoping exercise in seven European countries. PLoS One 7(4): e34188.

39. Regional Euthanasia Review Committees (2015) Netherlands Code of Practice in English.

40. Riley S (2017) Navigating the new era of assisted suicide and execution drugs. Journal of Law and the Biosciences 4(2): 424-434.

41. Edwards J (2013) Debating Euthanasia. Medical Law Review 21(4): 632641.

42. Alzheimer Europe (2009) Beneficence and non-maleficence.

43. Quaghebeur T, Dierckx de Casterlé B, Gastmans C (2009) Nursing and Euthanasia: A Review of Argument-Based Ethics Literature. Nurs Ethics 16(4): 466-486.

44. Munyaradzi M (2012) Critical reflections on the principle of beneficence in biomedicine. Pan Afr Med J 11: 29.

45. Medical Council of Hong Kong (2016) Code of Professional Conduct.

46. Mason Whitehead E (2008) Key Concepts in Nursing. SAGE publishing, UK.

47. O Brien JT, Burns A (2011) Clinical practice with anti-dementia drugs: a revised (second) consensus statement from the British Association for Psychopharmacology. J Psychopharmacol 25(8): 997-1019.

48. Rainer S (2014) Social Participation and Social Engagement of Elderly People. Procedia-Social and Behavioral Sciences 116(1): 780-785.

49. Entwistle V, Carter A, Cribb S, McCaffery M (2010) Supporting Patient Autonomy: The Importance of Clinician-patient Relationships. J Gen Intern Med 25(7): 741-745.

50. Grisso T, Appelbaum PS (1998) Assessing competence to consent to treatment: a guide for physicians and other health professionals. Oxford University Press, New York, USA.

51. Daroff RB, Jankovic J, Mazziotta JC, Pomeroy SL (2016) Bradley's Neurology in Clinical Practice ( $7^{\text {th }}$ edn.), Elsevier/Saunders Philadelphia, USA.

52. Meiner S (2015) Gerontologic Nursing ( $5^{\text {th }}$ edn.). Elsevier, USA.

53. Kim SYH (2011) The Ethics of Informed Consent in Alzheimer Disease Research. Nat Rev Neurol 7(7): 410-414.

54. Miller DG, Dresser R, Kim S (2018) Advance Euthanasia Directives: A Controversial Case and Its Ethical Implications. J Med Ethics 45(2): 8489.
55. Robinson C, Kolesar S, Boyko M, Berkowitz J, Calam B, (2012a) Awareness of do-not-resuscitate orders: What do patients know and want? Can Fam Physician 58(4): 229-233.

56. Itano J, Brant JM, Conde FA, Saria MG (2016) Core curriculum for oncology nursing $\left(5^{\text {th }}\right.$ edn.), Elsevier, St. Louis, USA.

57. Johnstone M (2016) Bioethics: A nursing perspective ( $6^{\text {th }}$ edn.), NSW: Elsevier, Chatswood, Australia.

58. Alkin MC (2011) Evaluation Essentials, First Edition: From A to Z (1 $^{\text {st }}$ edn.), The Guilford Press, New York, USA.

59. De Boer M, Hertogh C, Dröes R, Jonker C, Eefsting J (2010) Advance directives in dementia: Issues of validity and effectiveness. Int Psychogeriatr 22(2): 201-208.

60. Bolt E, Pasman H, Deeg D, Onwuteaka Philipsen B (2016) From Advance Euthanasia Directive to Euthanasia: Stable Preference in Older People? J Am Geriatr Soc 64(8): 1628-1633.

61. Olsen D (2016) Ethically Relevant Differences in Advance Directives for Psychiatric and End-of-Life Care. J Am Psychiatr Nurses Assoc 22(1): 5259.

62. Bravo G, Rodrigue C, Arcand M, Downie J, Dubois MF, et al. (2017) Nurses' perspectives on whether medical aid in dying should be accessible to incompetent patients with dementia: Findings from a survey conducted in Quebec, Canada. Geriatr Nurs 39(4): 393-399.

63. Porteri C (2018) Advance directives as a tool to respect patients' values and preferences: Discussion on the case of Alzheimer's disease. BMC Med Ethics 19(1): 9.

64. Solomon CG, Mitchell SL (2015) Advanced dementia. N Engl J Med 372(26): 2533-2540.

65. Bolt E, Snijdewind M, Willems D, Van Der Heide A, Onwuteaka Philipsen B (2015) Can physicians conceive of performing euthanasia in case of psychiatric disease, dementia or being tired of living? J Med Ethics 41(8): 592-598.

66. Hertogh C (2009) The role of advance euthanasia directives as an aid to communication and shared decision-making in dementia. J Med Ethics 35(2): 100-103.

67. Gaster B, Larson E, Curtis J (2017) Advance Directives for Dementia: Meeting a Unique Challenge. JAMA 318(22): 2175-2176.

68. Menzel P, Steinbock B (2013) Advance Directives, Dementia, and Physician-Assisted Death. J Law Med Ethics 41(2): 484-500.

69. Tsai C, Chuang C, Hung C, Lin C, Sung F, et al. (2014) Fracture as an Independent Risk Factor of Dementia: A Nationwide Population-Based Cohort Study. Medicine 93(26): 188.

70. Dean E (2017) Responding to a request to hasten death. Emerg Nurse 24(9): 11

71. Malpas P (2011) Advance directives and older people: Ethical challenges in the promotion of advance directives in New Zealand. J Med Ethics 37(5): 285-289.

72. Bradley SL, Woodman RJ, Tieman JJ, Phillips PA (2012) Use of advance directives by South Australians: results from the Health Omnibus Survey Spring. The Medical Journal of Australia 201(8): 467-469.

73. De Boer M, Dröes R, Jonker C, Eefsting J, Hertogh C (2011) Advance directives for euthanasia in dementia: How do they affect resident care in Dutch nursing homes? Experiences of physicians and relatives. J Am Geriatr Soc 59(6): 989-996

74. The Government of the Hong Kong Special Administrative Region (HKSAR) (2016) LCQ4: Euthanasia.

75. The Government of the Hong Kong Special Administrative Region (HKSAR) (2012) LCQ17: Euthanasia.

76. The Nursing Council of Hong Kong (2015) Code of Ethics and Professional Conduct for Nurses in Hong Kong. 